\title{
Information et publicité pour les médicaments s'adressant aux professionnels: les tribunaux appliquent le droit de manière stricte
}

\section{Valérie Junod*}

* Dr iur. (Genève),

J.S.M. (Stanford), LL.M. (UPenn).

Mes remerciements à Betty Junod et Tony Reynard pour leurs commentaires critiques ainsi qu'à

Hanspeter Kuhn et Max Giger pour leurs utiles suggestions.
Correspondance:

Dr iur. Valérie Junod, J.S.M., LL.M.

Université de Genève

Faculté de droit

Boulevard du Pont-d'Arve 40

CH-1211 Genève 4

valerie.junod@droit.unige.ch
La publicité pour les médicaments est un thème controversé qui ne cesse d'alimenter les débats au sein de la société. Elle suscite également des questions juridiques que les tribunaux suisses sont de plus en plus souvent appelés à trancher.

Depuis le $1^{\text {er }}$ janvier 2007, la Commission de recours pour les produits thérapeutiques (CORE PT) a cédé sa place au Tribunal administratif fédéral (TAF). Les décisions de l'Institut suisse des produits thérapeutiques (Swissmedic), et notamment celles appliquant les règles en matière de publicité, sont donc désormais attaquables devant le TAF. Créée en même temps que Swissmedic en 2002, la CORE PT avait atteint un rythme d'activité soutenu, avec 21 décisions publiées sur son site en 2006, 24 en 2005, pour seulement 23 de 2002 à 2004 [1].

Cet article analyse trois jugements rendus en 2006 par la CORE PT en matière de publicité destinée aux professionnels de la santé [2]. Ces jugements abordent deux questions importantes. Premièrement, comment distinguer les annonces publicitaires soumises à la réglementation fédérale des informations à caractère non promotionnel exclues du champ de la loi? Deuxièmement, comment déterminer si une publicité respecte les diverses précisions d'utilisation figurant dans l'autorisation de mise sur le marché (AMM) et dans l'information professionnelle (IP)? Des réponses apportées par la CORE PT à ces questions, il ressort que les exigences en matière de publicité sont de plus en plus strictes.

Cet article commence par exposer le contexte qui donne naissance à ces deux questions. Il examine ensuite comment la Commission de recours y a répondu. Le point suivant explique l'objectif que poursuivent tant Swissmedic que la CORE PT en restreignant les informations au sujet d'une utilisation dite off-label de médicaments. Finalement, l'article conclut par une critique de la situation actuelle où quasiment tout message positif relatif à de telles utilisations off-label est interdit.

\section{Contexte: les informations tirées de nouvelles études et leur communication aux médecins}

Même après avoir obtenu l'AMM initiale pour un nouveau médicament, l'entreprise pharmaceutique qui le commercialise en poursuit généralement l'étude. Ainsi, de nouvelles informations

\section{Information und Werbung}

für Medikamente an die Adresse von Fachleuten: Die Gerichte

\section{wenden das Gesetz strikte an}

Dieser Artikel diskutiert drei Entscheidungen, die die Eidgenössische Rekurskommission für Heilmittel im Bereich der Arzneimittelwerbung getroffen hat. Diese Entscheidungen betreffen zwei miteinander verbundene Fragen: Wann ist eine Aussage als Werbung anzusehen und fällt somit in den Anwendungsbereich der Verordnung über die Arzneimittelwerbung? Wann verletzt eine Werbebotschaft die Verordnung, weil sie die Grenzen, die die Zulassung und die Fachinformation festlegen, nicht einhält? Diese drei Entscheidungen sind die Gelegenheit für die Kommission, ihre Rechtsprechung zu präzisieren und zu verschärfen. Zunächst bestätigt die Kommission ihren sehr weitreichenden Begriff der Arzneimittelwerbung. Des weiteren schränkt sie die Möglichkeit der Pharmaunternehmen ein, zu ihren Gunsten die Ergebnisse von klinischen Versuchen, die nicht in der Zulassung oder der Fachinformation enthalten sind, zu verwerten. Die strikte Position der Kommission findet man ebenfalls in den letzten Richtlinien von Swissmedic zum Thema Werbung im Internet. 
scientifiques viennent régulièrement compléter les connaissances disponibles au moment de l'octroi de l'AMM. Toutefois, ces nouvelles informations ne sont pas nécessairement communiquées de suite à Swissmedic. Seules les informations négatives, c'est-à-dire celles révélant un risque ou un danger lié à l'emploi du médicament, doivent être communiquées sans délai à l'autorité en vue d'une mise à jour de l'AMM et/ou de l'IP (ci-après: AMM/IP) [3]. En revanche, la société pharmaceutique décide si et quand elle avise Swissmedic de nouvelles données positives (p.ex. une possible extension de l'indication thérapeutique, de la population pouvant être traitée, un changement de dosage ou de posologie). A supposer que ces données sont soumises à Swissmedic, celui-ci décide si elles sont suffisamment convaincantes, d'un point de vue scientifique et statistique, pour justifier la modification correspondante de l'AMM/IP.

Quand bien même l'entreprise n'a pas avisé Swissmedic de nouvelles données positives, elle peut vouloir les communiquer aux professionnels de la santé. Elle le fera par exemple par le biais d'un encart placé dans une revue médicale, l'encart reproduisant une petite partie de l'étude clinique publiée ayant conclu à ce nouveau bénéfice du médicament.

La question que l'autorité suisse se pose alors est de savoir si cette communication aux médecins relève de la publicité. Si oui, la communication est strictement réglementée par la Loi sur les produits thérapeutiques (LPTh) à ses articles 31 à 33 et par l'Ordonnance sur la publicité pour les médicaments (OPMéd). Swissmedic vérifie alors si son contenu respecte les exigences règlementaires, notamment si ce contenu est conforme aux données approuvées qui figurent dans l'AMM/IP. Si ce n'est pas le cas, l'entreprise concernée s'expose à une décision constatant la violation de l'article 5 alinéa 1 OPMéd et, éventuellement, à une sanction pénale sur la base de l'article 87 alinéa 1 lettre b LPTh.

\section{Quand est-ce qu'une communication relève de la publicité pour un médicament?}

Depuis sa décision de décembre 2005 [4], la CORE PT répète que le droit suisse retient une conception très large de la publicité [5]. N'importe quel message favorisant les ventes du médicament relève de la publicité [6]. Le but, et non la forme, du message est déterminant [7]. Ainsi, lorsque le message peut influencer - sous-entendu favorablement - la prescription du médicament par le médecin, il y a de fortes chances qu'il soit publicitaire. Selon la CORE PT, même un message purement informatif et objectif peut avoir cet effet, et donc tomber sous le coup des dispositions légales en matière de publicité [8]. Par exemple, la reprise sur le site Internet d'une société pharmaceutique d'un article publié dans une revue médicale par un chercheur tiers est qualifiée de publicitaire si le message véhiculé par l'article peut aboutir à une augmentation des ventes du médicament [9].

Pour échapper à cette qualification publicitaire, le message ne doit pas permettre l'identification d'un médicament particulier ou d'une classe particulière de médicaments [10]. En effet, selon l'article 1 alinéa 2 lettre c OPMéd, une information générale sur les maladies ne relève pas de la publicité. En pratique, rares sont toutefois les cas où les destinataires du message ne sont pas capables de «remonter» au médicament. La simple mention du nom scientifique de la substance active suffit à outrepasser cette limite [11].

\section{Quand est-ce qu'une publicité viole I'OPMéd?}

Une communication qualifiée de publicitaire viole l'OPMéd si elle contredit l'AMM/IP [12]. L'intérêt ou l'importance scientifique de la communication ne justifient pas une dérogation; seule une publication sans caractère publicitaire serait admissible. Autrement dit, l'entreprise pharmaceutique ne peut faire état des résultats favorables d'une étude si ceux-ci contredisent l'AMM/IP [13], et cela quand bien même la fiabilité de ce résultat serait incontestable. Si elle veut utiliser ce résultat dans une publicité, elle doit d'abord demander une modification de l'AMM/IP à Swissmedic [14]. Peu importe que le Code de promotion pharmaceutique de l'industrie pharmaceutique suisse semble tolérer des dérogations plus amples à ce principe [15]; pour la CORE PT, ces normes autorèglementaires ne la lient aucunement [16].

Pour déterminer si une publicité est conforme à l'AMM/IP, la CORE PT doit dégager le sens qu'il convient de donner à la publicité, respectivement à l'AMM/IP. A la publicité, elle attribue le sens que le destinataire moyen, et non pas la société pharmaceutique, peut raisonnablement lui donner [17]; le contexte global de la publicité est pris en compte. La CORE PT interprète également l'AMM/IP conformément au sens compris par ces professionnels, les éventuelles précisions apportées par Swissmedic étant prises en considération [18].

En application de ces principes, la CORE PT a confirmé dans les trois jugements examinés ici la décision de non-conformité prononcée par Swissmedic. 
Dans l'affaire HM 05.139, certaines modalités d'usage du pimecrolimus avaient été clarifiées par Swissmedic dans le numéro 4/2003 de son journal; la CORE PT a considéré ce complément d'information déterminant pour juger de la conformité de la publicité. Or, dans son contexte, le message publicitaire laissait entendre que le pimecrolimus pouvait être utilisé comme alternative aux corticostéroïdes, alors que Swissmedic l'avait classé comme traitement

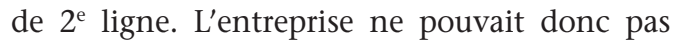
suggérer que le choix entre les deux médicaments ressortait simplement à l'appréciation des médecins.

Dans l'affaire HM 05.140, la CORE PT a estimé que l'allégué publicitaire «schnell» donnait aux médecins l'impression que les comprimés $\mathrm{T}$ de sumatriptan soulageaient plus vite les migraines que les usuels comprimés sécables. L'AMM/IP affirmant la bioéquivalence des deux formes susmentionnées, l'allégué publicitaire était donc contraire à l'OPMéd [19].

Dans l'affaire HM 05.124, la CORE PT a tranché que la recommandation d'un dosage élevé de quetiapine fumarate (jusqu'à $1600 \mathrm{mg}$ ) violait l'OPMéd puisque le dosage admis dans l'AMM/IP était de 800 mg maximum.

Sur ce même thème, on signalera encore l'arrêt Zambon/Dolo-Spedifem, dans lequel le Tribunal fédéral récuse des allégués publicitaires faisant état d'un effet antidouleur rapide et complet en moins de 30 minutes, alors que l'AMM/IP n'inclut que des données pharmacocinétiques indiquant une absorption moyenne en 30 minutes [20].

Pour tenter de légitimer des communications publicitaires qui outrepassent le cadre légal, les entreprises pharmaceutiques invoquent parfois la liberté d'expression. Systématiquement, l'argument est écarté. En Suisse, la liberté d'expression ne protège pas la publicité pharmaceutique - du moins pas davantage que la liberté économique, liberté considérée par les tribunaux comme d'application plus appropriée [21]. De surcroît, les restrictions légales apportées à cette liberté économique sont quasiment toujours jugées proportionnées au vu des intérêts publics en jeu [22].

Outre le respect de l'AMM/IP, il convient de mentionner deux autres exigences mises en évidence par la CORE PT. Dans l'affaire HM 05.124, la Commission a rappelé que, pour figurer dans une publicité, une étude doit, d'une part, être conforme aux bonnes pratiques d'essais cliniques (BCP) et, d'autre part, être publiée ou sur le point de l'être. A l'opposé, un "poster», un «abstract» ou un autre compte-rendu de nature semblable ne peut être cité en référence dans une publicité [23]. Dans l'affaire HM 05.139, la Commission a également exprimé sa réserve envers des études qui ne correspondent plus à l'état de la science. Ainsi, des résultats d'essais considérés comme dépassés en raison de nouvelles connaissances ne doivent plus utilisés à des fins publicitaires [24].

\section{Quels sont les objectifs de cette jurisprudence?}

En empêchant l'industrie pharmaceutique de vanter des bénéfices - même avérés - se trouvant en opposition avec les éléments de l'AMM/IP, la CORE PT et Swissmedic poursuivent le même objectif: contraindre l'entreprise pharmaceutique à soumettre à Swissmedic un dossier scientifique complet pour chaque extension d'utilisation que l'entreprise souhaite promouvoir. Grâce à ce dossier, Swissmedic est en mesure de réexaminer régulièrement les expériences acquises depuis l'autorisation initiale et d'actualiser l'AMM/IP du médicament. Il peut s'assurer que les avantages vantés sont dûment étayés par des données scientifiques issues d'essais cliniques valides. Médecins et patients bénéficient de cet examen de l'autorité, puisque la décision thérapeutique s'appuie alors sur une meilleure connaissance des bénéfices et des risques du médicament et cela pour une gamme plus étendue de conditions médicales.

A l'inverse, lorsque le médecin choisit - ou se trouve contraint, vu l'absence d'alternatives thérapeutiques - d'utiliser le médicament d'une manière qui n'est pas prévue par l'AMM/IP, il pénètre sur un terrain plus incertain. Quand bien même cet emploi du médicament hors indication («off-label use» en anglais) est légal et souvent conforme aux règles de l'art médical [25], il présente néanmoins des dangers. En effet, le médecin n'a pas l'assurance que les données auxquelles il se fie - souvent une étude clinique publiée - sont complètes et fiables [26]. Par exemple, il ne peut savoir si les résultats de cette étude sont représentatifs de ceux obtenus dans le cadre d'autres études effectuées sur le même médicament; un biais en faveur de la publication des études exhibant des résultats positifs peut fausser l'appréciation du médecin [27].

\section{Critique et conclusion}

Même si la conclusion à laquelle la CORE PT aboutit dans ces trois affaires ne prête pas le flan à la critique, on peut s'interroger sur la place à reconnaître aux études cliniques subséquentes à l'AMM. Lorsque de telles études débouchent sur des résultats significatifs, qu'ils soient positifs ou négatifs, il y a un intérêt légitime à les commu- 
niquer aux professionnels de la santé d'abord, voire ensuite aux médias, aux investisseurs et au public. Or, la CORE PT et Swissmedic excluent presque toute communication hors du canal des revues médicales.

On remarquera de manière incidente que, s'agissant plus particulièrement des non-professionnels de la santé (investisseurs, journalistes et grand public), Swissmedic a adopté en août 2006 une position rigoureuse - au demeurant critiquée par la doctrine juridique, en raison de l'absence de base légale suffisante [28] - qui restreint encore davantage la communication d'informations [29]. Cette directive délimite le type d'informations médicales qui peut figurer dans des sites Internet suisses, en fonction notamment des modalités d'accès au site par les différentes catégories d'utilisateurs. Swissmedic exige que des mots de passe permettent de réserver aux seuls professionnels de la santé l'accès aux pages web mentionnant des médicaments vendus sur prescription. On citera notamment le passage suivant qui traite de la «distinction entre les informations à l'intention des investisseurs et la publicité pour les médicaments»:

«Si le site présente des préparations ou des principes actifs nouveaux ou en cours d'élaboration, ou encore les perspectives futures et les priorités de l'entreprise dans le domaine de la recherche et du développement, il ne peut mentionner que la dénomination de la préparation, le nom du principe actif (denominatio communis internationalis), et le domaine thérapeutique ou le champ d'application. Les informations adressées aux investisseurs et aux analystes financiers peuvent renseigner ou avancer des prévisions sur le chiffre d'affaires, les parts de marché et les volumes de ventes, mais aucune déclaration plus précise quant à l'efficacité thérapeutique des médicaments ne peut être ajoutée.» [30]

Autrement dit, Swissmedic interdit la communication, sur sites Internet libres d'accès, d'informations discutant l'intérêt thérapeutique d'un médicament non approuvé. Il adhère ainsi pleinement à la définition extensive de la publicité qui veut qu'une information, même objective, relève de la publicité si elle peut augmenter les ventes du médicament [31].

On doit cependant se demander si cette position stricte défendue par Swissmedic et la CORE PT ne frustre pas, de manière excessive, l'indéniable besoin d'informations au sujet des médicaments. Pour le médecin qui cherche une information au sujet d'une possible utilisation off-label d'un médicament, les sources agréées de renseignements sont peu nombreuses; la principale demeure les revues médicales.

Un possible compromis serait de promouvoir davantage les plates-formes Internet recensant les études cliniques conformes aux GCP ( Good Clinical Practice»). Après bien des pressions, l'in- dustrie pharmaceutique s'est finalement engagée à mettre en place des registres d'essais cliniques. Ceux-ci inventorient aussi bien les opportunités de participer à un essai clinique en cours que les résultats des essais achevés, que ceux-ci aient ou non été soumis à l'autorité compétente [32]. Ces initiatives méritent d'être encouragées et mieux exploitées. On pourrait imaginer un «safe harbor» explicite (une tolérance légale) pour les sites Internet d'entreprises qui se bornent à répertorier, pour chaque médicament, les résultats de tous les essais cliniques [33]. Ainsi, le choix du médecin en faveur d'une utilisation off-label d'un médicament serait mieux éclairé, puisqu'il aurait accès à une information aussi complète et récente que possible.

\section{Références}

1 Tous les jugements de la Commission ne sont pas rendus publics. Même lorsque le jugement de la CORE PT est rendu public, il ne mentionne pas le nom de l'entreprise concernée. L'identité de celleci peut toutefois être inférée à partir des indications relatives au médicament qui figurent dans le texte du jugement. Quant aux décisions de Swissmedic en matière de publicité, elles ne sont jamais rendues publiques, même sous forme anonymisée.

2 Il s'agit des affaires: HM 05.124 du 7 mars 2006, HM 05.139 du 30 mai 2006 et HM 05.140 du 31 août 2006 (toutes trois en allemand). Ces trois jugements de la CORE PT n'ont pas - à ce jour du moins - été remis en cause par le Tribunal fédéral. En revanche, le Tribunal fédéral a confirmé trois jugements de la CORE PT concernant la publicité destinée au public. Il s'agit des affaires «Schering / Love Card» (arrêt 2A.20/2007 du 9 mai 2007), «Pfizer / Relpax» (arrêt 2A.63/2006 du 10 août 2006 confirmant le jugement HM 05.116) et «Zambon / Dolo-Spedifen» (arrêt 2A.607/2005 du 23 juin 2006 confirmant le jugement HM 05.109).

3 Bien que l'article 16 de l'Ordonnance sur les médicaments (OMéd; RS 812.212.21) ne le dise pas expressément, cette disposition ne s'applique qu'aux «faits et résultats nouveaux» qui affectent négativement la sécurité, l'efficacité ou la qualité du médicament.

4 Point 4.1 du jugement HM 05.116 du 14 décembre 2005 de la CORE PT. Voir aussi le jugement du 6 mai 2003, publié au JAAC 67.134.

5 L'article 2 lettre a OPMéd (RS 812.212.5) définit la publicité comme «toute forme d'information, de prospection ou d'incitation qui vise à encourager la prescription, la remise, la vente, la consommation ou l'utilisation de médicaments».

6 Le message doit s'adresser à un cercle large de personnes. Une communication faite à une personne en réponse à une demande spontanée formulée par celle-ci ne relève normalement pas de la publicité.

7 L'appréciation peut se faire de manière globale, en particulier si le message se décline sur plusieurs supports qui se renforcent les uns les autres (cf. l'arrêt du Tribunal fédéral dans l'affaire Schering, supra note 2, où l'offre d'un rabais était explicitée dans un communiqué sur Internet). Dans l'arrêt Pfizer / Relpax (supra note 2), le Tribunal fédéral a souligné qu'un message peut être qualifié de 
publicitaire, même lorsqu'il n'est pas établi que les ventes ont augmenté. Il a également suggéré que l'intention de l'auteur de la publicité ne serait pas déterminante, laissant toutefois la question ouverte, puisque dans l'affaire en cause la volonté publicitaire de Pfizer ne faisait pas de doute.

8 HM 05.116, point 4.1.

9 HM 05.124, points 4.2.1 et 4.3.2. HM 05.139, point 3.3.3. Voir la critique par Kramer B. Werbung oder Information? Zur Abgrenzung in der Arzneimittelwerbung, sic! 2007;489.

10 Voir l'arrêt Pfizer / Relpax, cité supra sous note 2 et le jugement ainsi confirmé de la CORE PT (HM 05.116). Voir également les commentaires de Schüpbach S. Arzneimittelwerbung: Migränebroschüre als unzulässige Publikumswerbung. Jusletter 2007.1.29 et de Eggenberger Stöckli U. Das Verbot von Publikumswerbung für verschreibungspflichtige Arzneimittel im Spannungsfeld zwischen Information und Werbung. Pharma Recht Schweiz. 2007;4:1.

11 HM 05.124, point 4.3.1. Voir aussi l'arrêt du Tribunal fédéral (ATF) $129 \mathrm{~V} 32$ dans l'affaire Pfizer / Viagra, ainsi que les explications et critiques par Kramer, supra note 9.

12 Bien entendu, une entreprise peut, et même doit (article 16 OMéd), communiquer immédiatement aux médecins des éléments qui relativisent la sécurité et l'efficacité de son médicament. Comme de telles informations ne vont pas conduire à une augmentation des ventes, il ne s'agit en principe pas d'une publicité soumise aux exigences de l'OPMéd.

13 La contradiction peut être implicite, par exemple lorsque la publicité porte sur une indication thérapeutique, une population de patients, un dosage ou une posologie non agréés par l'AMM/IP.

14 Cf. HM 05.139, point 3.1; HM 05.124, point 4.2.3; HM 05.140, points 3.1 et 3.2.4.

15 Voir les points 123.5 à 123.8, 131 et 133 du Code de bonnes pratiques de l'industrie pharmaceutique en Suisse du 4 décembre 2003.

16 Cf. HM 05.124, point 3.2.3.

17 La référence dans les trois affaires jugées par le CORE PT est le médecin moyen bien informé et de bonne foi. Cf. HM 05.139, points 3.2.1 et 3.2.2.

18 Ce principe paraît discutable s'il aboutit à faire primer le sens dégagé globalement par les professionnels sur le texte même de l'IP.

19 HM 05.140, point 3.2.1.

20 Arrêt 2A.607/2005, déjà cité sous note 2 supra. Cette publicité s'adressait au public, le médicament n'étant pas soumis à prescription.

21 Cf. HM 05.124, point 6 .

22 On saluera la position stricte de la CORE PT sur ce point. En effet, on voit mal comment la liberté d'expression pourrait disculper une publicité susceptible d'induire en erreur ou de créer la confusion auprès de ses destinataires. De surcroît, les bénéfices liés au maintien du contrôle préalable des médicaments par l'autorité justifient l'interdiction pour les entreprises d'utiliser à leur profit des études qui n'ont pas été soumises à ce contrôle. A cet égard, les tribunaux américains qui ont accepté d'entrer en matière sur de tels arguments ont ouvert une regrettable boîte de Pandore. Cf. Henney JE. Safeguarding Patient Welfare: Who's In Charge? Ann
Intern Med. 2006;145:305-7; Shuchman M. Drug Risks and Free Speech - Can Congress Ban Consumer Drug Ads? N Engl J Med. 2007;356:2236-9.

23 Cf. HM 05.124, point 4.1.3.

24 HM 05.139, point 3.3.1.

25 Le médecin doit faire preuve d'une précaution accrue lorsqu'il prescrit un médicament hors indication. Il est notamment censé en informer son patient. Un groupe d'expert composé de représentants de l'Association des pharmaciens cantonaux suisses et de Swissmedic a émis des Recommandations au sujet de l'utilisation des médicaments au sens de l'«off label use» (24 juillet 2006).

26 Par exemple, dans l'affaire HM 05.139 (point 3.3.1), la CORE PT exprime ses doutes sur l'indépendance des auteurs d'études sponsorisées par l'industrie, quand bien même ces études satisfont aux exigences formelles de l'art. 5 al. 5 OPMéd. Elle s'appuie sur un article de Delaney B. Is society losing control of the medical research agenda? BMJ. 2006; 332:1063-4.

27 Les registres de résultats d'essais cliniques alimentés par les entreprises pharmaceutiques aident à surmonter ce biais (par exemple le portail sur le site de la Fédération internationale de l'industrie du médicament [FIIM/IFMPA] ou les sites individuels d'entreprises). Toutefois, ces registres sont certainement moins souvent consultés que les revues médicales.

28 Cf. Bürgi H. Richtlinien der Swissmedic zur Arzneimittelwerbung im Internet - kritische Bemerkungen. PJA. 2007;1:70-8. Dans son arrêt «Schering» (cons. 8) cité supra sous note 2, le Tribunal fédéral laisse ouverte la question de savoir si l'observation de cette directive, s'agissant de l'exigence d'un mot de passe, est seule à garantir l'impunité au regard de la loi.

29 Journal Swissmedic 8/2006, p. 802 et «questions réponses» du 22 décembre 2006.

30 Journal Swissmedic 8/2006, p. 803, mise en évidence ajoutée.

31 A mon avis, les restrictions d'accès extrêmement rigides (mots de passe obligatoires) que la directive de Swissmedic impose frustrent une soif d'information, parfois légitime, des patients. Ces personnes se tourneront naturellement vers des sources étrangères, en particulier des sites Internet non suisses. Ces sites n'étant soumis à aucun contrôle de Swissmedic (et insuffisamment contrôlés par d'autres autorités étrangères), on peut se demander si le résultat obtenu silence quasi total sur les sites médicaux en .ch et pléthore d'informations parfois contradictoires et douteuses sur les sites en .com est bien dans l'intérêt des patients suisses.

32 Voir les différents documents sur le site créé par la FIIM à http://clinicaltrials.ifpma.org. Voir également la plate-forme d'enregistrement d'essais cliniques en cours sur le site de l'Organisation mondiale de la santé (OMS) et sa liste des registres associés. En revanche, l'OMS n'a pas encore mis au point son registre de résultats d'essais cliniques.

33 Pour que l'information ne soit pas biaisée, ces sites devraient lister tant les études sponsorisées par l'entreprise que celles sponsorisées par des tiers. Les études répertoriées devraient être accompagnées d'un résumé objectif en «plain English». Les sites en question devraient pouvoir avoir un nom de domaine avec extension .ch. 